\title{
Calculation Method for Flight Limit Load of V-band Clamp Separation Shock*
}

\author{
Takashi IWASA $^{* *}$ and Qinzhong SHI ${ }^{* * *}$ \\ ${ }^{* *}$ Tottori University, \\ 4-101 Koyama-cho Minami, Tottori, JAPAN \\ E-mail: iwasa@mech.tottori-u.ac.jp \\ ***Japan Aerospace Exploration Agency, \\ 2-1-1 Sengen, Tsukuba, Ibaraki, JAPAN
}

\begin{abstract}
A simplified calculation method for estimating a flight limit load of the V-band clamp separation shock was established. With this method, the flight limit load is estimated through addition of an appropriate envelope margin to the results acquired with the simplified analysis method proposed in our previous paper. The envelope margin used in the method was calculated based on the reviews on the differences observed between the results of a pyroshock test and the analysis. Using the derived envelope margin, a calculating formula of the flight limit load, which envelopes the actual pyroshock responses with a certain probability, was developed. Based on the formula, flight limit loads for several actual satellites were estimated and compared to the test results. The comparative results showed that the estimated flight limit loads appropriately envelope the test results, which confirmed the effectiveness of the proposed method.
\end{abstract}

Key words: Simplified Calculation Method, Envelope Margin, Flight Limit Load, V-band Clamp Separation Shock

\section{Introduction}

V-band clamp separation devices have been frequently used in the separation process of a spacecraft from a launch vehicle. This separation process imposes severe pyroshock loadings to the spacecraft interface ${ }^{(1,2)}$, because the firing from a pyrotechnic device generates a shock load of high magnitude and high frequency. Therefore, estimation of such severe pyroshock loadings on a spacecraft interface in the early design stages is essential to provide a necessary input for the determination of the pyroshock environment applied to sensitive electromechanical equipments and to establish the associated shock design requirements which are necessary to ensure the overall spacecraft reliability.

In our previous paper ${ }^{(3)}$, a simplified analysis method for predicting the Shock Response Spectrum (SRS) at the spacecraft interface induced by the V-band clamp separation shock was established. This method applies the single degree of freedom (D.O.F) model to make the calculation easy for the SRS. Figure 1 indicates the comparison on the SRS given by the simplified analysis and the actual pyroshock test. Acc1 - Acc4 in the figure indicate the test results measured at the near field of the V-band clamp separation devices. This figure shows that the analysis results roughly envelope the test results, while some test results exceed the analysis results in the high frequency range. This indicates that an appropriate margin be necessary to make estimation on the reliable flight limit load of V-band clamp separation shock.

${ }^{*}$ Received 27 Jan., 2010 (No. 10-0041) [DOI: 10.1299/spacee.3.15]

Copyright $@ 2010$ by JSME 


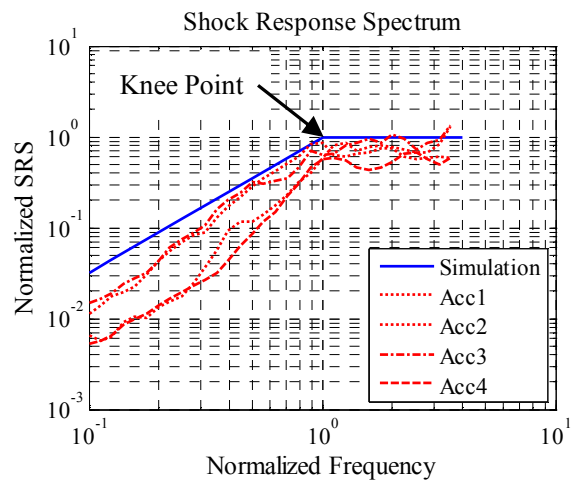

Fig. 1 Simulation Result of the Simplified Analysis Method

In this paper, an appropriate envelope margin was studied with the purposes of predicting the reliable flight limit load of the V-band clamp separation shock. Firstly, the envelope margin was formulated based on the differences observed between the results of a pyroshock test and the simplified analysis. After that, the calculation formula of the simplified calculation method to estimate the flight limit load was developed through the incorporation of the formulated envelope margin into the simplified analysis method. Then, the appropriate envelope margin was specifically calculated based on the statistical analysis on the differences obtained from the simplified analysis for the pyroshock tests on several actual satellites. Through application of the simplified calculation method, which now has the derived envelope margin adopted, to actual satellites, their flight limit loads were estimated and compared to their test results. Based on the results, the validity and availability of the proposed method were discussed.

\section{Simplified Calculation Method for Flight Limit Load}

\subsection{Outline of the Simplified Analysis Method proposed in Our Previous Paper}

In the simplified analysis method proposed in our previous paper ${ }^{(3)}$, the SRS due to V-band clamp separation shock was calculated from the response of the single D.O.F model, which represents the dominating pyroshock response of the spacecraft interface. Then, the major vibration mode of the spacecraft interface after the separation of the V-band clamp separation devices was assumed to be in the circular form so that the single D.O.F model can be applied.

Since the SRS magnitude given by the dominating pyroshock response generally appears as the knee point in the SRS figure, the simplified analysis method focuses on the prediction of the knee point of the SRS due to V-band clamp separation shock. When SRS envelope curve is configured based on the calculated knee point, two straight lines are used. The gradient of the straight line drawn in the frequency range lower than the calculated knee point is set to be 6-11dB/OCT, and the other in the higher frequency range is set to be $0 \mathrm{~dB} / \mathrm{OCT}$. The outline of the SRS envelope curve given by the simplified analysis method is shown in Fig.1. Figures 2 and 3 depict an analysis model of the spacecraft interface.

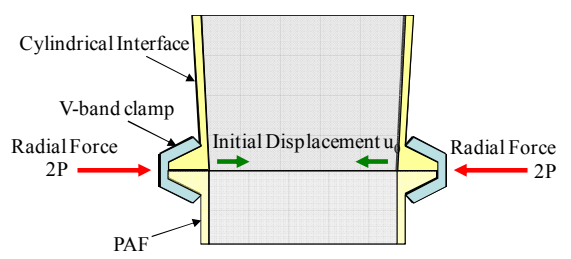

Fig. 2 Depiction of the Spacecraft Interface 


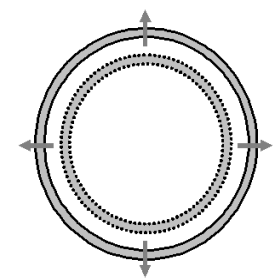

Fig. 3 Assumed Vibration Mode

\subsection{Relationship between Pyroshock Test Results and Analysis Results}

As stated in the previous subsection 2.1, since the simplified analysis method focuses on the prediction of the knee point in the SRS, this method enables no calculations on the SRS magnitude except the knee point. Therefore, as shown in Fig. 1, the actual SRS magnitude at the high frequency range may be higher than the calculated SRS envelope. This phenomenon occurs due to the fact that the assumed vibration mode with the circular form essentially does not represent the dispersion of the actual pyroshock responses, which exist along the circumference of the interface.

Therefore, the relationship between the SRS magnitude measured on the spacecraft interface in the actual pyroshock test and that acquired through analysis is expressed in the following equation.

$$
S R S_{\text {test }}^{i}=\mu_{\text {test }}+\delta_{\text {test }}^{i}=S R S_{\text {sim }}+\delta_{\text {sim }}+\delta_{\text {test }}^{i}
$$

Here, $\mu_{\text {test }}$ indicates the average of the SRS magnitudes measured on the circumference of the interface in the actual pyroshock test, which is given by the following equation.

$$
\mu_{\text {test }}=\frac{1}{N} \sum_{i=1}^{N} S R S_{\text {test }}^{i}
$$

where, $N$ is the total number of the measurement points on the circumference of the interface in each satellite, and $i$ indicates the measurement point. $\delta_{\text {test }}^{i}$ in Eq.(1) represents the dispersion of the SRS magnitude at each measurement point from the average $\mu_{\text {test }}$. Namely, it is calculated in the following equation.

$$
\delta_{\text {test }}^{i}=S R S_{\text {test }}^{i}-\mu_{\text {test }}
$$

$\delta_{\text {sim }}$ in Eq.(1) is the difference between the SRS magnitude calculated through the simplified analysis method and the average of the SRS magnitudes measured on the circumference of the interface. Therefore, it is formulated as follows.

$$
\delta_{\text {sim }}=\mu_{\text {test }}-S R S_{\text {sim }}
$$

\subsection{Calculation Formula of Flight Limit Load of V-band Clamp Separation Shock}

Equation (1) shows that the SRS magnitude measured on the spacecraft interface in the actual pyroshock test is represented by the analysis results $S R S_{\text {sim }}$ and two differences $\delta_{\text {sim }}$ and $\delta_{\text {test }}^{i}$. Therefore, when these two differences are obtained from the results of several pyroshock tests and analyses, the appropriate envelope margin that is incorporated 
into the analysis results can be calculated through the statistical analysis on these two differences. This means that the calculation formula of the flight limit load to envelop the actual pyroshock responses with a certain probability can be derived based on the analysis results and the derived envelope margin incorporated. Accordingly, the calculation formula of the appropriate envelope margin was discussed based on the relationship given by Eq.(1).

Generally, it is reported that the distribution characteristic of the SRS magnitudes measured on the random points on the spacecraft follows the log-normal distribution ${ }^{(1)}$. Therefore, when the SRS magnitudes are transformed onto the logarithmic scale, the distribution characteristics of the two differences $\delta_{\text {sim }}$ and $\delta_{\text {test }}^{i}$ are also considered to follow the normal distribution. This is because these two differences were derived from the SRS magnitudes measured on the spacecraft interface and the analysis.

When the logarithmic scale of the SRS magnitude is given by the following equation,

$$
y=\log _{10}(S R S)
$$

the two differences $\bar{\delta}_{\text {sim }}$ and $\bar{\delta}_{\text {test }}^{i}$, which are transformed onto the logarithmic scale, are formulated as follows.

$$
\begin{gathered}
\bar{\delta}_{\text {sim }}=\bar{\mu}_{\text {test }}-y_{\text {sim }} \\
\bar{\delta}_{\text {test }}^{i}=y_{\text {test }}^{i}-\bar{\mu}_{\text {test }}
\end{gathered}
$$

where,

$$
\bar{\mu}_{\text {test }}=\frac{1}{N} \sum_{i=1}^{N} y_{\text {test }}^{i}
$$

Then, based on the assumption that the distribution characteristics of these differences follow the normal distribution, the standard deviation of the logarithmic variance $\bar{\delta}_{\text {test }}^{i}$ that indicates the dispersion from the average is calculated in the following equation.

$$
\bar{\sigma}_{\text {test }}=\sqrt{\frac{1}{P-1} \sum_{k=1}^{P}\left(\bar{\delta}_{\text {test }}^{k}\right)^{2}}
$$

Where, $P$ indicates the total number of measurement points on the circumference of the interfaces in all the satellites. In the same way, the average and the standard deviation of the logarithmic difference $\bar{\delta}_{\text {sim }}$ that is a difference between the analysis results and the average of the measured SRS are calculated from the following equations.

$$
\begin{aligned}
& \bar{\mu}_{s i m}=\frac{1}{M} \sum_{j=1}^{M} \bar{\delta}_{\text {sim }}^{j} \\
& \bar{\sigma}_{\text {sim }}=\sqrt{\frac{1}{M-1} \sum_{j=1}^{M}\left(\bar{\delta}_{\text {sim }}^{j}-\bar{\mu}_{\text {sim }}\right)^{2}}
\end{aligned}
$$


Here, $M$ indicates the total number of pyroshock tests. From the results and the relationship given by Eq.(1), the calculation formula of the flight limit load of the V-band clamp separation shock, which envelopes the actual pyroshock responses with a certain probability, is expressed as follows.

$$
y_{\text {flight }}=y_{\text {sim }}+\bar{\mu}_{\text {sim }}+k_{n \beta \gamma} \sqrt{\bar{\sigma}_{\text {sim }}^{2}+\bar{\sigma}_{\text {test }}^{2}}
$$

Here, the envelope margin applied to the simplified analysis results is formulated as follows.

$$
\alpha=\bar{\mu}_{s i m}+k_{n \beta \gamma} \sqrt{\bar{\sigma}_{\text {sim }}^{2}+\bar{\sigma}_{\text {test }}^{2}}
$$

$k_{n \beta \gamma}$ in Eqs.(12) and (13) is generally called the normal tolerance factor, and is applied for the calculation of the one-sided (upper-tail) normal tolerance limit, which is defined as the value of the SRS magnitude that exceeds at least $\beta$ portion of all the possible values of the SRS magnitudes with a confidence of $\gamma$ (this probability is generally called $P \beta / \gamma$ in space engineering $\left.{ }^{(2)}\right) . n$ in the subscript of $k_{n \beta \gamma}$ indicates the number of data. Since the flight limit load calculated from Eq. (12) is on the logarithmic scale, the flight limit load on the linear scale can be finally given by the following equation.

$$
S R S_{\text {flight }}=10^{y_{\text {flight }}}
$$

\subsection{Physical Meaning of the Proposed Calculation Formula}

The physical meaning of Eq. (12) can be explained as follows. Firstly, the simplified analysis results are modified with the use of the average variance $\bar{\mu}_{\text {sim }}$, which is given by Eq. (10) . This process makes the analysis results closer to the average of the SRS magnitudes measured on the circumference of the interface in the actual pyroshock test. After that, the appropriate deviation $k_{n \beta \gamma} \sqrt{\bar{\sigma}_{\text {sim }}^{2}+\bar{\sigma}_{\text {test }}^{2}}$ is added to the modified analysis results for the estimation on the flight limit loads that envelope the actual pyroshock responses with the probability $P \beta / \gamma$.

Figure 4 depicts the physical meaning of the calculation formula of Eq.(12). In this figure, the envelope margin is set constant by conducting a statistical analysis on all the differences appeared in the higher frequency range (cf. Statistical Analysis Area in Fig.4). In this paper, all adopted the envelope margin were derived in the same way as represented in Fig. 4.

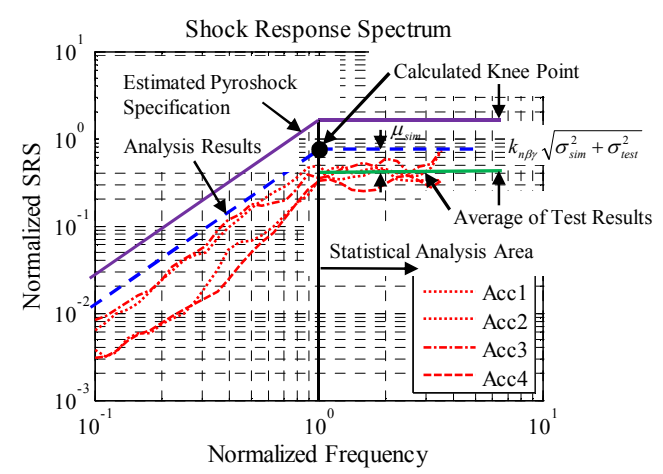

Fig. 4 Physical Meaning of the Calculating Formula in Eq.(12) 


\section{Calculation of Envelope Margin used in the Proposed Calculation Method}

\subsection{Outline of the Calculation}

Since the calculation formulas of the flight limit load of the V-band clamp separation shock were derived in the previous section, the appropriate envelope margin was specifically calculated through a statistical analysis on the two differences observed in the test data measured in the actual pyroshock tests and the analysis results on three satellites. The total number of pyroshock tests intended for the calculation added up to eight ( $M=8$ ). Those tests included the pyroshock tests conducted for the structure model and the proto-flight model of those satellites. The measurement points in each satellite were installed in the near-field from the V-band clamp separation devices. Although the number of measurement points in each satellite was different, at least more than four points were used. The total number of measurement points in all the satellites was forty three $(P=43)$.

\subsection{Distribution Characteristics of the Differences $\delta_{\text {sim }}, \delta_{\text {test }}^{i}$}

It was firstly investigated whether the distribution of the SRS magnitudes at the spacecraft interface, which are used in this paper, followed the log-normal distribution or not. Figure 5 indicates the normal probability plots of the SRS magnitudes given by the actual pyroshock tests and by the analysis. These SRS magnitudes were transformed onto the logarithmic scale. The figure shows that the logarithmic SRS magnitudes are distributed along the straight lines, and the distribution of the logarithmic SRS magnitudes roughly follows the normal distribution ${ }^{(1,4)}$. Accordingly, when the SRS magnitudes are transformed onto the logarithmic scale, the distribution characteristics of the two differences $\bar{\delta}_{\text {sim }}$ and $\bar{\delta}_{\text {test }}^{i}$, which are calculated in Eqs. (6) and (7), will also follow the normal distribution. This means that Eqs.(8) - (14) in the previous section can be applied for the calculation of the appropriate envelope margin.

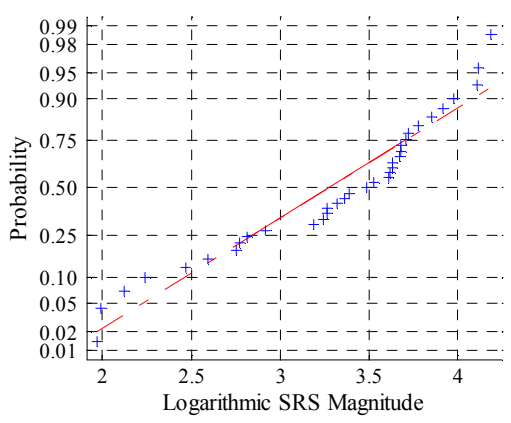

(a) Pyroshock Test Results

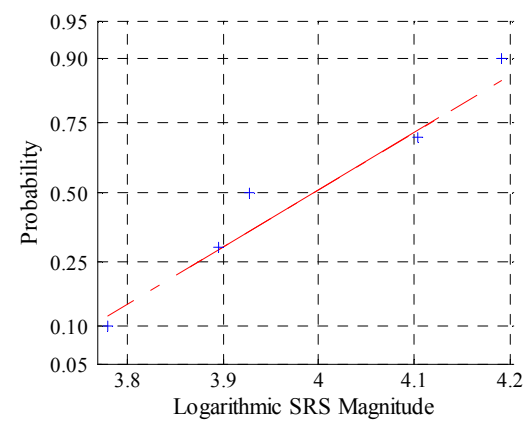

(b) Analysis Results

Fig. 5 Normal Probability Plot of Log. SRS

\subsection{Derivation of Appropriate Envelope Margin $\alpha$}

Table 1 indicates the average and the standard deviation of the two logarithmic differences $\bar{\delta}_{\text {sim }}$ and $\bar{\delta}_{\text {test }}^{i}$. These results were calculated from Eqs.(9), (10) and (11) with the application of the actual pyroshock test results and analysis results on three satellites.

Tab. 1 Average and Standard Deviation of Logarithmic Differences, $\bar{\delta}_{\text {sim }}, \bar{\delta}_{\text {test }}^{i}$

\begin{tabular}{|c|c|c|}
\hline \multicolumn{2}{|c|}{$\bar{\delta}_{\text {sim }}$} & $\bar{\delta}_{\text {test }}$ \\
\hline $\bar{\mu}_{\text {sim }}$ & $\bar{\sigma}_{\text {sim }}$ & $\bar{\sigma}_{\text {test }}$ \\
\hline-0.167 & 0.060 & 0.169 \\
\hline
\end{tabular}


Table 2 indicates the envelope margin calculated in Eq.(13) based on the results in Tab.1. These results also represent the logarithmic scale. In Tab.2, the values under P95/50 and P99/90, which are often used as the tolerance factors for AT level and QT level respectively in the spacecraft engineering, are listed.

From the result, the logarithmic flight limit load of the V-band clamp separation shock can be easily acquired from Eq.(12), which is finally transformed onto the linear scale through Eq.(14).

Tab. 2 Envelope Margin $\alpha$ (Logarithmic Scale)

\begin{tabular}{|c|c|}
\hline P95/50 (AT Level) & P99/90 (QT Level) \\
\hline$k_{\infty 95 / 50}$ & $k_{\infty 99 / 90}$ \\
\hline 0.127 & 0.251 \\
\hline
\end{tabular}

\section{Results and Discussion}

\subsection{Validation on the Simplified Calculation Method}

Validity of the calculation formula of the flight limit load was investigated through the comparison on the estimated results and the pyroshock test results. Here, the pyroshock test results used for the study are the same as the test data applied to the derivation of the envelope margin in the previous section. Therefore, when the calculation formula and the envelope margin were appropriately derived, the estimated flight limit load in this study would envelop the test results with a certain probability.

Figure 6 indicates the comparison on the results of the calculation and the tests where various degrees of tension were applied to the V-band clamp separation devices on the same satellite. Ordinates in these figures indicate the SRS magnitudes normalized by the maximum of the analysis results. Abscissas indicate the frequencies normalized by the knee frequency calculated through the analysis. The gradient of the SRS curve in the lower frequency range was set at $9 \mathrm{~dB} / \mathrm{OCT}$. From the figure, it turned out that the estimated flight limit load for P99/90 (QT level) appropriately enveloped the actual pyroshock responses of the V-band clamp separation shock, while one part of the test results exceeded the estimated results for P95/50 (AT level). These results indicated that the estimated flight limit load appropriately enveloped the test results with a certain probability.

Figure 7 indicates the comparison on the results of the calculation and the tests, where pyroshock tests were conducted upon different satellites. Ordinates in these figures indicate the SRS magnitudes normalized by the maximum of the analysis results. Abscissas indicate the frequencies normalized by the knee frequency calculated through the analysis. The gradient of the SRS curve in the lower frequency range was set at $9 \mathrm{~dB} / \mathrm{OCT}$. From the figures, it turned out that the estimated flight limit load for P95/50 (AT level) as well as for P99/90 (QT level) appropriately enveloped the actual pyroshock responses induced by the V-band clamp separation shock. Therefore, these results also indicated that the estimated flight limit load appropriately enveloped the test results for the different satellites.

In conclusion, these results confirmed the validity of the calculation formula derived in the previous section. 


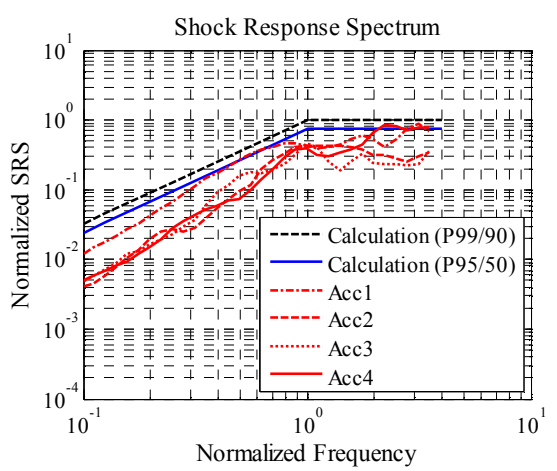

(a) Lower Tension of the V-band Clamp

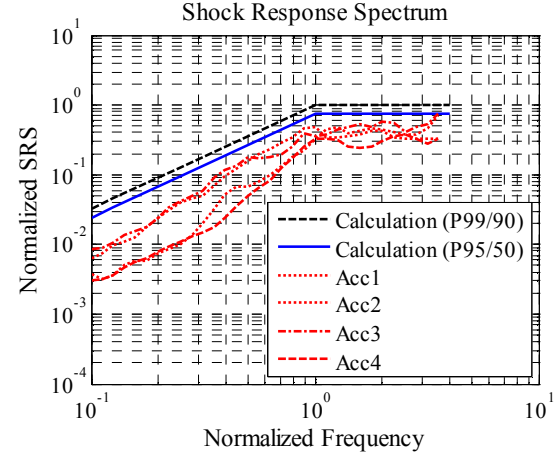

(b) Higher Tension of the V-band Clamp

Fig. 6 Comparative Results on Satellite 1

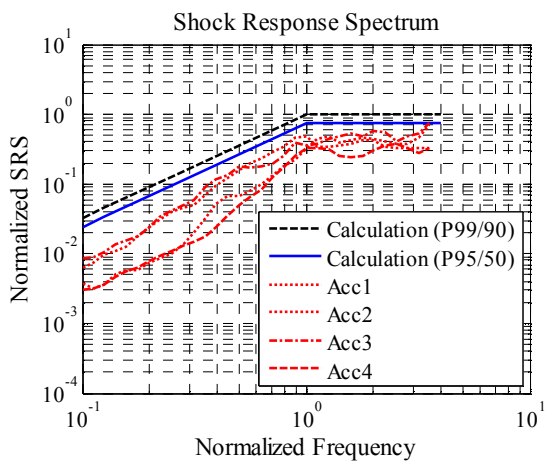

(a) Satellite 1

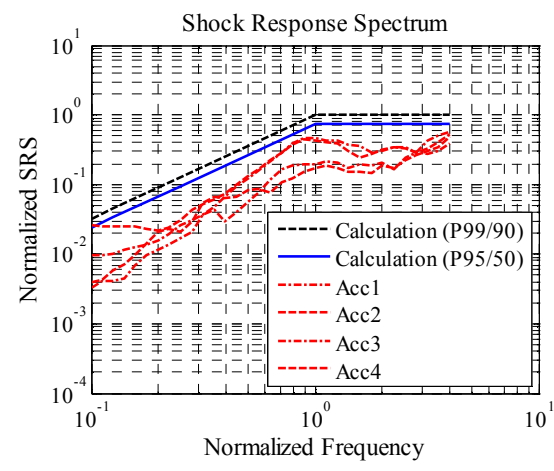

(b) Satellite 2

Fig. 7 Comparative Results on Satellite 1 and Satellite 2

\subsection{Availability on the Simplified Calculation Method}

The availability of the simplified calculation method was investigated through the comparison on the estimated flight limit load and the pyroshock test results for newly-developed satellites.

Figure 8 indicates the comparison on the results of the calculation and the tests. Ordinates in the figures indicate the SRS magnitudes normalized by the maximum of the analysis results. Abscissas indicate the frequencies normalized by the knee frequency calculated through the analysis. The gradient of the SRS curve in the lower frequency range was set at $9 \mathrm{~dB} / \mathrm{OCT}$. From the figures, it turned out that the estimated flight limit load for P99/90 (QT level) appropriately enveloped the actual pyroshock responses induced by the V-band clamp separation shock for the newly-developed satellites, while one part of the test results exceeded the estimated results for P95/50 (AT level). These results indicate that the estimated flight limit load appropriately enveloped the test results with a certain probability. Therefore, the availability of the simplified calculation method was confirmed for the newly-developed satellites. 


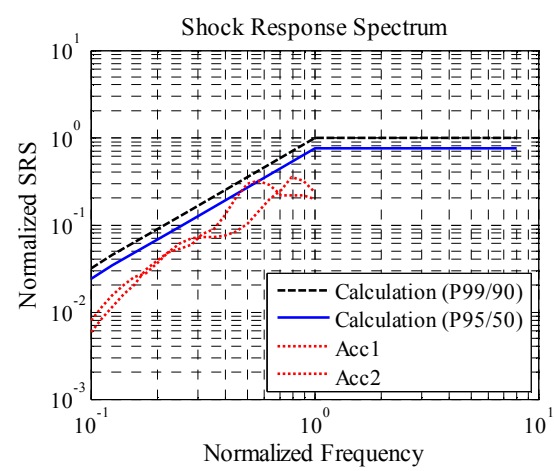

(a) Satellite 1

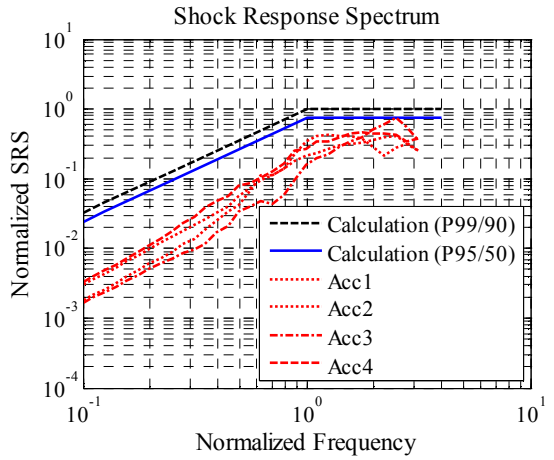

(b) Satellite 2

Fig. 8 Comparative Result on Newly-Developed Satellite

\section{Conclusion}

A simplified calculation method for estimating a flight limit load of the V-band clamp separation shock was investigated based on the simplified analysis method proposed in our previous paper. The conclusions obtained from the study are summarized below.

1. The appropriate envelope margin to be incorporated into the results derived from the simplified analysis method established in our previous paper was formulated based on the reviews on the differences observed between the results of the pyroshock tests and the analysis.

2. A calculation formula to estimate the flight limit load enveloping the actual pyroshock responses with a certain probability was developed based on a simplified analysis method and the formulated envelope margin.

3. The appropriate envelope margin was specifically derived from the statistical analysis on the differences obtained from the simplified analysis for the pyroshock tests on three actual satellites.

4. Through the comparison of the flight limit load estimated through the proposed calculation method and the test results, it was observed that the estimated flight limit load appropriately enveloped the actual pyroshock responses with a certain probability. Those results proved the validity and availability of the proposed method.

\section{References}

(1) "Pyroshock Test Criteria," NASA-STD-7003, May 18, 1999.

(2) "Dynamic Environmental Criteria," NASA HDBK-7005, March 13, 2001.

(3) T. IWASA and Q. SHI, "Simplified Estimating Method for Shock Response Spectrum Envelope of V-band Clamp Separation Shock," Journal of Space Engineering, JSME, Vol. 1, No. 1, 2008, pp.-46-57.

(4) Normann L. Johnson, Fred C. Leone, "Statistics and Experimental Design in Engineering and the Physical Sciences Volume 1," John riliy \& Sons, Inc., 1997.

(5) "Test Requirements for Space Vehicles," MIL-STD-1540B. 